\title{
Independent Component Analysis for Magnetic Resonance Image Analysis
}

\author{
Yen-Chieh Ouyang, ${ }^{1}$ Hsian-Min Chen, ${ }^{1}$ Jyh-Wen Chai, ${ }^{2,3,4}$ Cheng-Chieh Chen, ${ }^{1}$ Clayton Chi-Chang Chen, ${ }^{4,5}$ \\ Sek-Kwong Poon, ${ }^{6}$ Ching-Wen Yang, ${ }^{7}$ and San-Kan Lee ${ }^{8}$ \\ ${ }^{1}$ Department of Electrical Engineering, National Chung Hsing University, Taichung 402, Taiwan \\ ${ }^{2}$ Department of Radiology, College of Medicine, China Medical University, Taichung 404, Taiwan \\ ${ }^{3}$ School of Medicine, National Yang-Ming University, Taipei 112, Taiwan \\ ${ }^{4}$ Department of Radiology, Taichung Veterans General Hospital, Taichung 407, Taiwan \\ ${ }^{5}$ Department of Medical Imaging and Radiological Science, Central Taiwan University of Science and Technology, \\ Taichung 406, Taiwan \\ ${ }^{6}$ Division of Gastroenterology, Department of Internal Medicine, Center of Clinical Informatics Research Development, \\ Taichung Veterans General Hospital, Taichung 407, Taiwan \\ ${ }^{7}$ Computer Center, Taichung Veterans General Hospital, Taichung 407, Taiwan \\ ${ }^{8}$ Chia-Yi, Veterans Hospital, Chia-Yi 600, Taiwan
}

Correspondence should be addressed to Clayton Chi-Chang Chen, ccc@mail.vghtc.gov.tw

Received 11 October 2007; Revised 21 December 2007; Accepted 30 December 2007

Recommended by Chein-I Chang

Independent component analysis (ICA) has recently received considerable interest in applications of magnetic resonance (MR) image analysis. However, unlike its applications to functional magnetic resonance imaging (fMRI) where the number of data samples is greater than the number of signal sources to be separated, a dilemma encountered in MR image analysis is that the number of MR images is usually less than the number of signal sources to be blindly separated. As a result, at least two or more brain tissue substances are forced into a single independent component (IC) in which none of these brain tissue substances can be discriminated from another. In addition, since the ICA is generally initialized by random initial conditions, the final generated ICs are different. In order to resolve this issue, this paper presents an approach which implements the over-complete ICA in conjunction with spatial domain-based classification so as to achieve better classification in each of ICA-demixed ICs. In order to demonstrate the proposed over-complete ICA, (OC-ICA) experiments are conducted for performance analysis and evaluation. Results show that the OC-ICA implemented with classification can be very effective, provided the training samples are judiciously selected.

Copyright (c) 2008 Yen-Chieh Ouyang et al. This is an open access article distributed under the Creative Commons Attribution License, which permits unrestricted use, distribution, and reproduction in any medium, provided the original work is properly cited.

\section{INTRODUCTION}

One of the greatest challenges in magnetic resonance (MR) image analysis is feature extraction of clinical information to be used for medical diagnosis. Unlike most medical modalities, the MRI is developed using tissue parameters such as spin-lattice (T1) and spin-spin (T2) relaxation times and proton density $(\mathrm{PD})$ to characterize various tissue information at the same anatomical area [1]. As a result, the features extracted from MR images can be obtained by spatial domain-based information as well as tissue characterization information derived from different pulse sequences. There- fore, an effective feature extraction technique should take advantage of both types of information.

Over the past years, MR images are processed from two different perspectives. One is a traditional and general approach which considers MR images as multidimensional data so that multivariate analysis can be applied. For example, in most applications MR images are processed as 3dimenaional (3D) image cube with pixels replaced by voxels so that image processing techniques such as segmentation, region growing, classification, and pattern recognition are readily applied $[2,3]$. In particular, a recent classificationbased transform, called eigenimaging filter, has shown 
success in producing a composite image for feature extraction [4-9]. Nevertheless, the information provided by tissue characterization resulting from different pulse sequences is still not fully explored for image analysis. In order to address this issue, another approach views MR images as an image sequence that can be treated as multispectral images [10-12] where each band image can be considered as an image acquired by a particular pulse sequence. In light of multispectral images, the tissue characterization can be explored via different pulse sequences. Several recent works based on linear mixture analysis were reported [13-16]. This paper presents a new approach that combines multispectral analysis with spatial domain-based classification techniques so that multispectral and spatial information can be fully explored by a statistical independency-based transform, called independent component analysis (ICA) and feature extraction-based classification techniques.

ICA has shown great promise in functional magnetic resonance imaging (fMRI) which is a method that provides functional information of MR images in time series as a temporal function [17]. Recently, a new application of ICA in MR image analysis was investigated by Nakai et al. in [18]. Compared to what has been done for fMRI, ICA applications to MR images have yet to be explored. A major difference between $\mathrm{PMRI}$ and MR image analysis is the mixing matrix used in the ICA for blind signal source separation. Since the samples for fMRI are collected along a temporal sequence, the number of samples, denoted by $L$, is usually greater than the sources to be separated, denoted by $p$; the ICA used for fMRI is generally under-complete in the sense that the ICA deals with under representation of a mixed model. In this case, the ICA intends to solve an over-determined system with $L>p$ consisting $L$ equations specified by the number of samples with signal sources to be separated as $p$ unknowns. As a result, there was generally no solutions. On the other hand, the samples used for MR image analysis are actually a stack of images acquired by different pulse sequences specified by three magnetic resonance parameters: spin-lattice (T1) and spin-spin (T2) relaxation times and proton density (PD). In this case, only three images can be acquired for image analysis. If the number of signal sources to be separated, $p$, is greater than the number of different combinations of pulse sequences, $L$, the ICA becomes an under-determined system with $L<p$ where the ICA must deal with an overcomplete representation of a mixed model. In this case, there are many solutions. As a result, fMRI and MR image analysis are completely different applications and the approaches developed for one application cannot be directly applied to another. However, for ICA to be implemented as undercomplete ICA, Nakai et al. assumed that the number of sensors, $L$, is greater than or equal to the number of sources, $p$, where the sensor is an MR imaging system; the number of sensors corresponds to the combinations of acquisition parameters echo time (TE) and repetition time (TR), and a signal source is represented by a tissue cluster characterized by a unique combination of T1, T2 relaxation times and PD. This key assumption makes the ICA under-complete with $L>p$ so that traditional ICA approach can be readily applied. Using the changes in signal intensity of each tissue cluster re- flected by combinations of TR and TE before and after the ICA transform, the contrast resulting from effects of the ICA can be used to perform image evaluation for a particular tissue such as white matter (WM) and gray matter (GM).

Unfortunately, Nakai et al.'s ICA approach overlooked an important issue. If we interpret the number of pulse sequences used in MR acquisition, denoted by $L$ and tissue substances such as water, blood, fat, GM, WM, cerebral spinal fluid (CSF), and muscle, as signal sources to be separated, denoted by $p$, the $L$ is actually less than $p$. As a consequence, the problem to be solved is an under-determined system with $L<p$, where the ICA must deal with an over-complete representation of a mixed model. This is completely opposite to Nakai et al.'s ICA approach as well as most ICA-based approaches used for $\mathrm{AMRI}$, since there are many solutions for the over-complete ICA (OC-ICA) as opposed to no solutions for the under-complete ICA (UC-ICA). Interestingly, using the OC-ICA for MR image analysis has not been explored.

More specifically, the idea of the OC-ICA can be interpreted by a well-known pigeon-hole principle in discrete mathematics. We assume that a spectral band image such as an image pulse sequence as a pigeon hole and the brain substances as pigeons flying into pigeon holes. In light of this interpretation, $L$ and $p$ represent the number of pigeon holes and number of brain substances to be classified, respectively, where one spectral band can be used to accommodate one brain substance. So, when $L<p$, it implies that there are more pigeons than pigeon holes. In this case, at least one pigeon must accommodate more than one pigeon. That is, if there are two or more pigeons accommodated in a pigeon hole, it indicates that a spectral band cannot be used to discriminate two or more brain substances. This illustrates the major issue encountered in MR image analysis, and the ICA to be dealt with is the OC-ICA, where the number of image pulse sequences used for acquisition is generally smaller than the number of brain substances of interest.

Additionally, there are two major issues resulting form the implementation of the ICA needed to be addressed from MR image analysis. For the ICA to produce independent components (ICs), an initial condition is required to initialize an ICA algorithm. A general approach is to randomly generate unit vectors to be used as initial projection vectors which can converge to a final set of projection vectors to produce ICs. The problem with such a random approach is that the final sets of projection vectors produced by two different sets of random initial projection vectors are generally different. As a result, the ICA implemented by the same user in different times or two different users at the same time will produce different sets of projection vectors to produce completely different sets of ICs. Such inconsistency undermines repeatability of the ICA and makes the ICA unstable. Besides, due to the use of random initial projection vectors the order that the ICs are generated is completely random and does not necessarily indicate the significance or importance of an IC. In other words, an IC generated earlier does not necessarily imply that it is more important than the one generated later. Consequently, image evaluation cannot be performed until all ICs are generated. Most importantly, since the representation of a mixing model used by the ICA is 
over-complete, there are no sufficient ICs to accommodate brain tissue substances in addition to the WM, GM, and CSF. Namely, many single ICs can be used to separate more than one signal source so that there is no unique solution to select which IC is the best for particular signal source. What is worse is that due to use of random initial projection vectors brain tissue substances are also forced to be randomly mixed in different ICs. These two reasons, that is, many solutions for the OC-ICA and the use of random initial projection vectors, are exactly the cause of inconsistent ICs in final results. For example, the WM, GM, and CSF may be randomly accommodated in a single IC as will be demonstrated in our experiments in this paper. Under such a circumstance, there is no best way to select a single IC to discriminate these three brain tissue substances one from another. This inevitable phenomenon is caused by the use of random initial projection vectors by the ICA and the lack of ICs resulting from the inherent nature in the OC-ICA. In order to resolve this dilemma, this paper develops a new approach which implements the OC-ICA in conjunction with classification where a feature extraction-based classifier is included as a post-OC-ICA processing technique to perform classification. Two well-known classifiers, Fisher's linear discriminant analysis (FLDA) and support vector machine (SVM), are used for this purpose because they both have been shown as most effective and promising classification techniques in pattern recognition. Surprisingly, experimental results show that with the help of classification, the OC-ICA performs significantly better in terms of classification of three major brain tissue substances: WM, GM, and CSF. Despite that the threeclass classification may appear in different orders resulting from a random order in which ICs are generated, such a random appearing order has very little effect on classification results. In other words, the results produced by the OC-ICA with classification are nearly independent of random initial projection vectors. This advantage is very useful and valuable since it frees a user from using random initial projection vectors to initialize an ICA algorithm.

\section{INDEPENDENT COMPONENT ANALYSIS}

The key idea of the ICA assumes that data are linearly mixed by a set of separate independent sources and these signal sources can be demixed according to their statistical independency measured by mutual information. In order to validate its approach, an underlying but very crucial assumption is that at most one source in the mixture model can be allowed to be a Gaussian source. This is due to the fact that a linear mixture of Gaussian sources is still a Gaussian source. More precisely, let $\mathbf{x}$ be a mixed signal source vector expressed by

$$
\mathbf{x}=\text { As, }
$$

where $\mathbf{A}$ is an $L \times p$ mixing matrix and $\mathbf{s}$ is a $p$-dimensional signal source vector with $p$ signal sources needed to be separated. Two scenarios are of interest in implementing the ICA. One is the case that the mixing matrix $\mathbf{A}$ in (1) has more dimensions than it requires for blind signal separation, that is,
$L>p$. In this scenario, the ICA has few bases (i.e., signal sources) than the samples provided (i.e., observations in the observable vector $\mathbf{x}$ ) and thus referred to as under-complete ICA which implies that the ICA has under-representative bases. However, according to system theory, the linear system equation described by (1) is actually an over-determined system, in which case there exits no solution to (1). In order to resolve this dilemma, a Dimensionality Reduction (DR) is generally used to reduce dimensionality of the mixing matrix A from $L$ to $p$ to make (1) is solvable. On the other extreme, if (1) has fewer samples than the sources to be demixed, that is, $L<p$, the ICA is called over-complete, referred to as OC-ICA which implies that it has over-representative bases to solve an under-determined system for (1). As a consequence, there are many solutions to (1) and there is no way to select best ICs to perform classification. Interestingly, there is very little work reported about how to cope with the OC-ICA, particularly how to address the issues caused by insufficient ICs and the use of random initial projection vectors which result in inconsistent ICs. However, due to the nature of the OC-ICA only a limited number of ICs is available to be used for signal source separation. When the number of signal sources is greater than the number of ICs, some of ICs are forced to accommodate more than one signal source in which case there is no way to a particular IC to characterize signal sources. Additionally, the use of random initial projection vectors also causes random mixtures of signal sources as well as noise in each of ICs. Unfortunately, such severe disadvantages have been overlooked and never been addressed effectively in the past.

\section{OC-ICA WITH CLASSIFICATION}

In order to mitigate the issue that more than one signal source accommodated in a single IC, a feature extractionbased classification technique is included as a post OC-ICA processing technique to classify substances of interest. Since the WM, GM, and CSF are of major interest in MR image classification, three ICs produced by $\mathrm{PD}, \mathrm{T} 1$, and $\mathrm{T} 2$ can be used to accommodate and classify these three substances. However, because of random initial conditions each IC may be randomly mixed by different brain tissue substances. The introduced follow-up classification technique can remove undesired substances from ICA-generated ICs while retaining the substances of interest. Although different mixtures of the WM, GM, and CSF may appear in different orders due to random orders that ICs are generated, the experiments conducted in this paper show that their classification results produced by different sets of random initial projection vectors will be nearly the same.

Two well-known feature extraction-based classification techniques, Fisher's linear discriminant analysis and support vector machine, are developed in this paper to be implemented in conjunction with the OC-ICA as a post OCA-ICA processing technique. This selection was based on the fact that these two techniques have been shown very effective in pattern classification and both are designed by feature extraction criteria. 


\subsection{Fisher's linear discriminant analysis (FLDA)}

The Fisher's linear discriminant analysis (FLDA) is one of the most widely used pattern classification techniques in pattern recognition [19] and was also used for feature extraction [9]. Its strength in pattern classification lies on the criterion used for optimality, which is called Fisher's ratio defined by the ratio of between-class scatter matrix to within-class scatter matrix.

More specifically, assume that there are $n$ training sample vectors, $\left\{\mathbf{r}_{i}\right\}_{i=1}^{n}$ for $p$-class classification, $C_{1}, C_{2}, \ldots, C_{p}$ with $n_{j}$ being the number of training sample vectors in the $j$ th class $C_{j}$. Let $\boldsymbol{\mu}$ be the global mean of the entire training sample vectors, denoted by $\boldsymbol{\mu}=(1 / n) \sum_{i=1}^{n} \mathbf{r}_{i}$, and let $\boldsymbol{\mu}_{j}$ be the mean of the training sample vectors in the $j$ th class $C_{j}$, denoted by $\boldsymbol{\mu}_{j}=\left(1 / n_{j}\right) \sum_{\mathbf{r}_{i} \in C_{j}} \mathbf{r}_{i}$. The within-class scatter matrix, $\mathbf{S}_{W}$, between-class scatter matrix $\mathbf{S}_{B}$, and total scatter matrix are defined in [19] as follows,

$$
\begin{aligned}
\mathbf{S}_{W} & =\sum_{j=1}^{p} \boldsymbol{S}_{j}, \quad \text { where } \boldsymbol{S}_{j}=\sum_{\mathbf{r} \in C_{j}}\left(\mathbf{r}-\boldsymbol{\mu}_{j}\right)\left(\mathbf{r}-\boldsymbol{\mu}_{j}\right)^{T}, \\
\mathbf{S}_{B} & =\sum_{j=1}^{p} n_{j}\left(\boldsymbol{\mu}_{j}-\boldsymbol{\mu}\right)\left(\boldsymbol{\mu}_{j}-\boldsymbol{\mu}\right)^{T} \\
\mathbf{S}_{T} & =\sum_{i=1}^{n}\left(\mathbf{r}_{i}-\boldsymbol{\mu}\right)\left(\mathbf{r}_{i}-\boldsymbol{\mu}\right)^{T}=\mathbf{S}_{W}+\mathbf{S}_{B} .
\end{aligned}
$$

By virtue of (2) and (3), Fisher's ratio (also known as Rayleigh's quotient [19]) is then defined by

$$
\frac{\mathbf{x}^{T} \mathbf{S}_{B} \mathbf{x}}{\mathbf{x}^{T} \mathbf{S}_{W} \mathbf{x}} \text { over vector } \mathbf{x} \text {. }
$$

The goal of the FLDA is to find a set of feature vectors that maximize Fisher's ratio specified by (5). The number of feature vectors found by Fisher's ratio is determined by the number of classes, $p$, to be classified, which is $p-1$.

\subsection{Support vector machine (SVM)}

In addition to the FLDA, another classification-based discriminant function, called Support Vector Machine (SVM) [20] can be also used as a post OC-ICA processing technique. The SVM is designed to find an optimal hyperplane that separates two classes of data samples as farther as possible by maximizing the margin of separation between classes and the hyperplane. It is originally developed as a binary classifier. A salient difference that the SVM is different from other classifiers is the use of training samples. The SVM uses and incorporates only a few so-called confusing data samples, referred to as slack variables, in its optimization problems to maximize the margin of separation among these samples. Another crucial and unique feature that the SVM has is the data space on which they perform. The SVM makes use of a nonlinear kernel to map the original data space into a higher dimensional space to resolve the issue of linear inseparability. Since the details of SVM can be found in many references in [20], we only briefly review its approach as follows.
The SVM was originally developed by Vapnik based on statistical learning theory [21]. Consider a two-category classification problem with a given set of training data $\left\{\left(\mathbf{r}_{i}, d_{i}\right)\right\}_{i=1}^{n}$, where $\left\{\mathbf{r}_{i}\right\}_{i=1}^{n}$ are $n$ samples with their associated binary decisions $\left\{d_{i}\right\}_{i=1}^{n}$ which are specified by either +1 or -1 . Assume that an SVM is specified by a linear discriminate function given by $g(\mathbf{r})=\mathbf{w}^{T} \mathbf{r}+b$, where $\mathbf{w}$ is a weight vector and $b$ is a bias. More specifically, given a set of training data, $\left\{\left(\mathbf{r}_{i}, d_{i}\right)\right\}_{i=1}^{n}$, an SVM finds a weight vector $\mathbf{w}$ and bias $b$ that satisfy

$$
d_{i}= \begin{cases}+1 & \text { if } \mathbf{w}^{T} \mathbf{r}_{i}+b \geq 0 \\ -1 & \text { if } \mathbf{w}^{T} \mathbf{r}_{i}+b<0\end{cases}
$$

and maximize the margin of separation defined by distance between a hyperplane and closest data samples. In particular, (6) can be rederived by incorporating its binary decision into discriminant function as follows:

$$
d_{i}\left(\mathbf{w}^{T} \mathbf{r}_{i}+b\right) \geq 1 \quad \text { for } 1 \leq i \leq n .
$$

For a linear separable problem, the SVM attempts to position a class boundary so that the margin from the nearest example is maximized. According to (7), the distance $\rho$ between a sample vector $\mathbf{r}$ and its projected vector on the hyperplane $g(\mathbf{r})=\mathbf{w}^{T} \mathbf{r}+b=0$ is specified by $\rho=g(\mathbf{r}) /\|\mathbf{w}\|$ with $\mathbf{w}$ being the normal vector of the hyperplane. Since $g(\mathbf{r})$ takes only +1 or -1 , the distance $\rho$ is then defined by

$$
\rho= \begin{cases}1 /\|\mathbf{w}\| & \text { if } d_{i}=+1, \\ -1 /\|\mathbf{w}\| & \text { if } d_{i}=-1 .\end{cases}
$$

Using (8), we define the margin of separation between two classes, denoted by $\rho$, as $\rho=2 /\|\mathbf{w}\|$. By virtue of (6)-(8), the SVM is to find an optimal weight vector $\mathbf{w}$ minimizing

$$
\Phi(\mathbf{w})=(1 / 2) \mathbf{w}^{T} \mathbf{w}=(1 / 2)\|\mathbf{w}\|^{2}
$$

subject to constraints specified by (7)

An optimal solution to the above optimization problem is given by

$$
\begin{gathered}
\mathbf{w}^{\mathrm{SVM}}=\sum_{i=1}^{n} \alpha_{i}^{\mathrm{SVM}} d_{i} \mathbf{r}_{i}, \\
1=d_{s}=\left(\mathbf{w}^{\mathrm{SVM}}\right)^{T} \mathbf{r}^{s}+b \Longrightarrow b=1-\left(\mathbf{w}^{\mathrm{SVM}}\right)^{T} \mathbf{r}^{s},
\end{gathered}
$$

with $\mathbf{r}^{s}$ is a support vector on the hyperplane with its decision $d_{s}=+1$.

Figure 1 illustrates the concept of the SVM where two classes of data sample vectors determined by (6) are denoted by $\Omega^{+}$and $\Omega^{-}$consisting of "open circles" and "crosses", respectively, and the vectors satisfying the equality of (7) are called support vectors.

The SVM discussed above was developed to separate two classes which are linearly separable. That is, the data sample vectors in two classes can be separated by a distance greater than $\rho$ from the hyperplane shown in Figure 1. However, in many applications, such desired situation may not occur. In other words, some data sample vectors fall in the region 


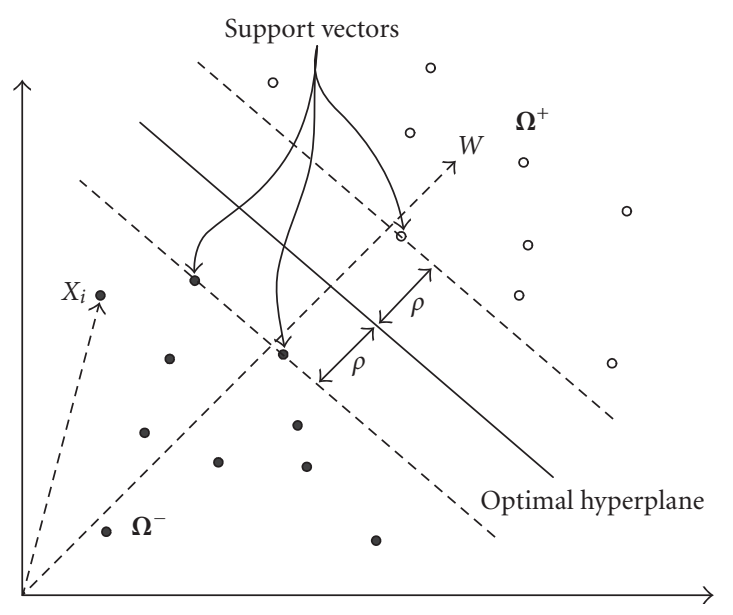

FIGURE 1: Illustration of SVM.

within the distance less than $\rho$ from the hyperplane or even on the wrong side of the hyperplane. These data sample vectors can be considered to be either bad or confusing data sample vectors and they cannot be linearly separated. In this case, the SVM developed for linear separable problems outlined by (6)-(10) must be rederived to take care of such confusing data sample vectors. In doing so, a new set of positive parameters, denoted by $\left\{\xi_{i}\right\}_{i=1}^{n}$ and referred to as slack variables, must be introduced to measure the deviation of a data sample vector from the ideal condition of linear separability, in which case $\xi_{i}<0$. However, if $0 \leq \xi_{i} \leq 1$, the ith data sample vector $\mathbf{x}_{i}$ falls in within the region with distance less than margin of separation but on the correct side of the decision surface specified by the hyperplane. On the other hand, if $\xi_{i}>1$, the $i$ th data sample vector $\mathbf{x}_{i}$ falls on the wrong side of its decision surface. In light of the mathematical interpretation, these issues can be addressed by the following inequalities:

$$
\begin{aligned}
d_{i}\left(\mathbf{w}^{T} \mathbf{r}_{i}+b\right) & \geq 1-\xi_{i}, \quad \text { for } 1 \leq i \leq n, \\
\xi_{i} & \geq 0, \quad \text { for } 1 \leq i \leq n .
\end{aligned}
$$

By incorporating (11) into the object function, $\Phi(\mathbf{w})$ in (9) can be modified as

$$
\Phi(\mathbf{w})=(1 / 2) \mathbf{w}^{T} \mathbf{w}+C \sum_{i=1}^{n} \xi_{i}, \quad \text { with } C>0
$$

By means of (11)-(12), a linear nonseparable problem can be solved by the SVM (for more details about the SVM, see [20]).

\section{EXPERIMENTS}

Two sets of experiments were conducted to substantiate the utility of our proposed OC-ICA with classification in MR image analysis and to demonstrate its advantages over the traditional ICA. One is MR brain synthetic images available on website [22] and the other is real MR brain images obtained in the Taichung Veterans General Hospital.

\subsection{Synthetic brian image experiments}

The synthetic images to be used for experiments in this section were the axial $\mathrm{T} 1, \mathrm{~T} 2$, and proton density $\mathrm{MR}$ brain images (with 5-mm section thickness, $0 \%$ noise, and $0 \%$ intensity nonuniformity) resulting from the MR imaging simulator of McGill University, Montreal, Canada (http://www.bic.mni.mcgill.ca/brainweb). The image volume provided separates volumes of tissue classes, such as CSF, GM, WM, bone, fatness, and background. The use of these web MR brain images is to allow researchers to reproduce our experiments for verification. Figures $2(a)-2(c)$ show three MR brain images with specifications provided in [22] where Figure 2(a) is acquired by the proton density modality with slice_thickness $=5 \mathrm{~mm}$, noise $=0 \%$, INU (intensity nonuniformity) $=0 \%$, Figure 2 (b) is acquired by the T1 modality with slice_thickness $=5 \mathrm{~mm}$, noise $=0 \%$, INU $=0 \%$, and Figure $2(\mathrm{c})$ is acquired by the T2 modality with slice_thickness $=5 \mathrm{~mm}$, noise $=0 \%$, INU $=0 \%$. Figure 3 provides the ground truth which is also available on website [22] for brain tissue substances in the images in Figure 2. This ground truth will be used to verify the results obtained for our experiments.

In order to implement supervised FLDA and SVM, four classes were considered, WM, GM CSF, and image background (BKG), for classification. For each class, 20 training samples were marked by dark points in the GM, CSF, WM images and bright points in the BKG image in Figure 4. These samples were selected according to prior knowledge provided in Figure 3 where the outside of brain skull was considered as the BKG.

Since the FastICA uses random initial projection vectors, the final results of ICs are generally different. In order to demonstrate this phenomenon, the FastICA was implemented three times for the three MR brain images in Figure 2 and their results are shown in Figures 5(a), 6(a), and 7(a) as three scenarios where the three ICs in these three scenarios are not only different but also appear in different orders. The three ICs in each scenario were then stacked one atop another to form a new 3-IC stacked image cube used for FLDA classification with results shown in Figures 5(b), 6(b), and 7(b), and SVM classification with results shown in Figures 5(c), 6(c), and 7(c).

According to the above three scenarios in Figures 5-7, the three ICs in each scenario were mixed differently by three major substances, WM, GM, and CSF. For example, the IC1 in Figure 5(a) was badly mixed by the three substances and IC1 in Figure 6(a) was heavily mixed by the GM and CSF. Scenario 3 in Figure 7(a) was the best scenario which could separate the GM, WM, and CSF reasonably well. To resolve these two issues, the FLDA and SVM were applied to 3IC stacked image cubes formed by the three ICs in Figures $5(\mathrm{a}), 6(\mathrm{a})$, and $7(\mathrm{a})$ of the three scenarios and their results are shown in Figures 5(b) and 5(c), 6(b) and 6(c), and 7(b) and 7(c). Surprisingly, the FLDA and SVM significantly improved classification results where WM, GM, and CSF were 


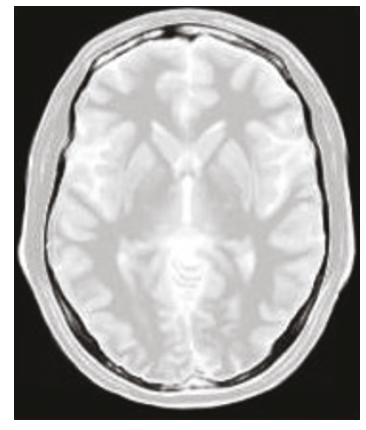

(a) $\mathrm{PD}$

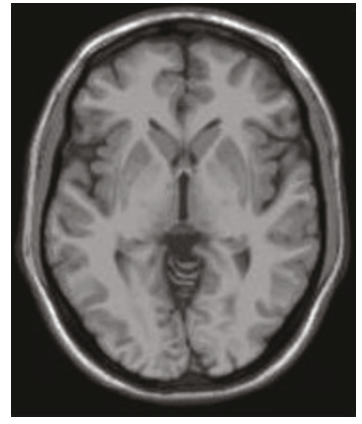

(b) T1

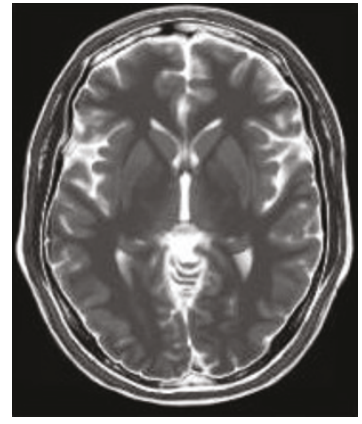

(c) $\mathrm{T} 2$

Figure 2: Three MR brain images.

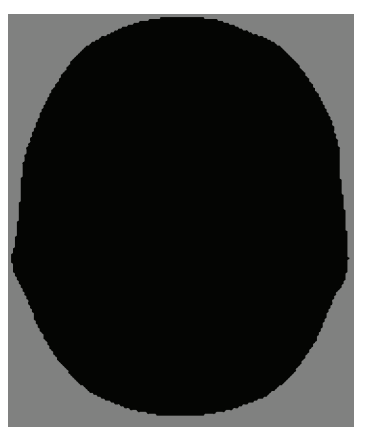

Background

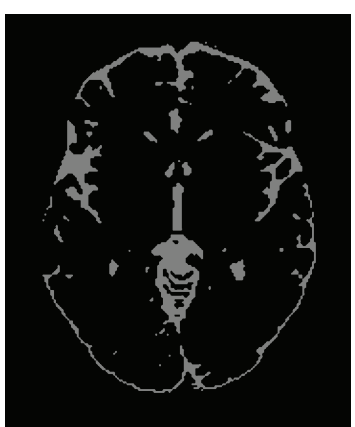

CSF

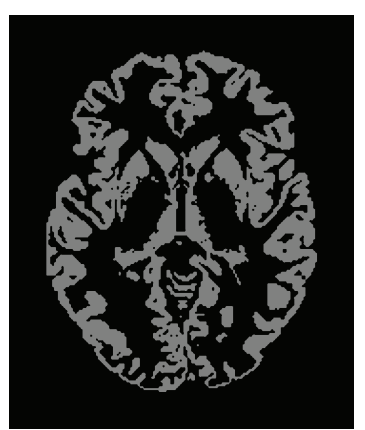

GM

(a)

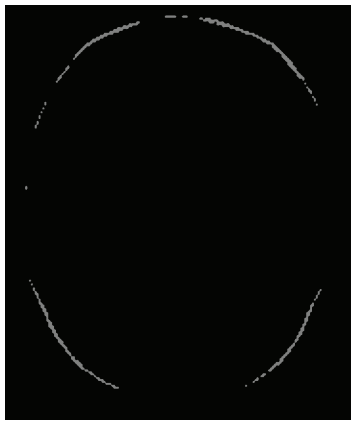

Fat

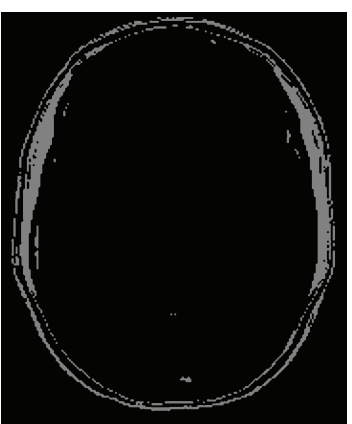

Muscle/skin

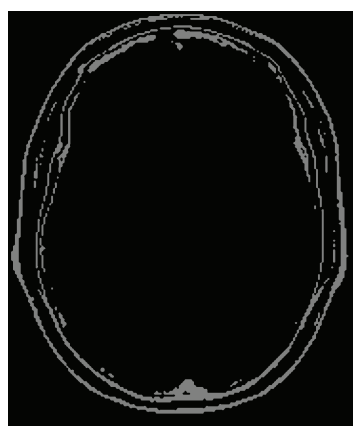

Skin

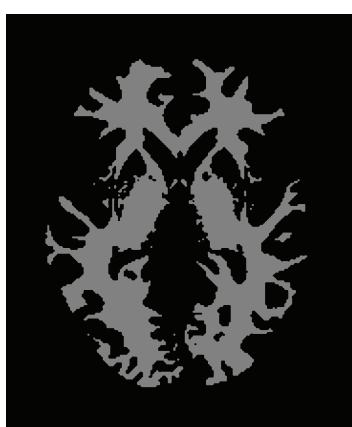

WM

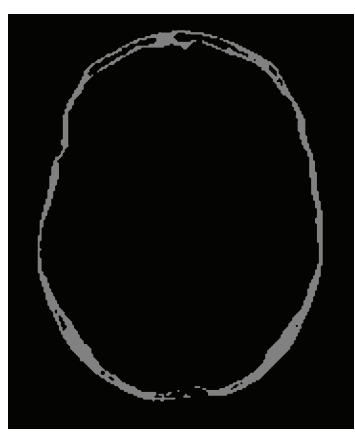

Skull

(b)

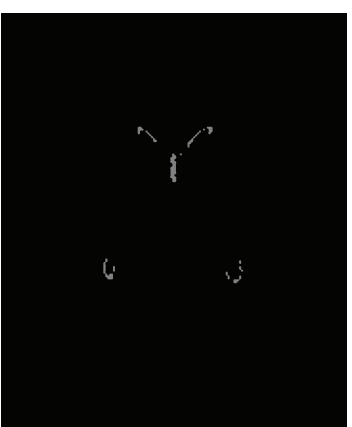

Glial matter

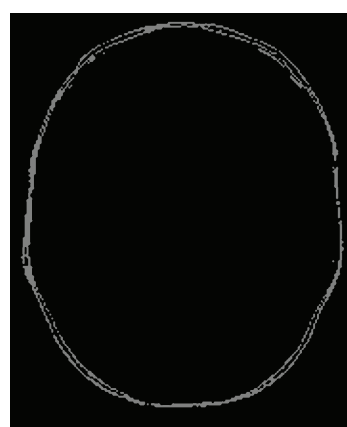

Connective

(c)

FIgURE 3: Ground truth of brain tissue substances for images in Figure 2. 


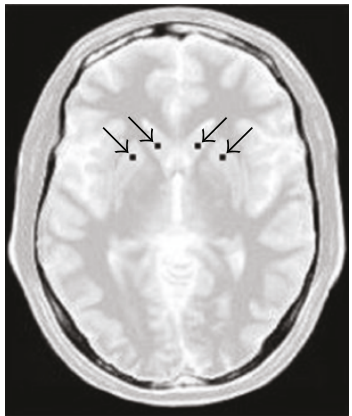

GM

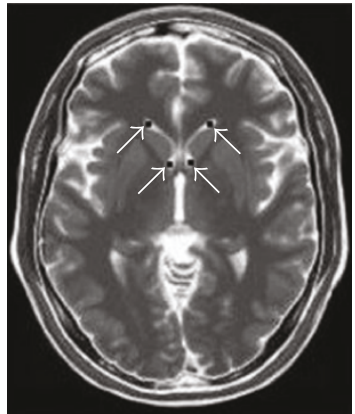

CSF

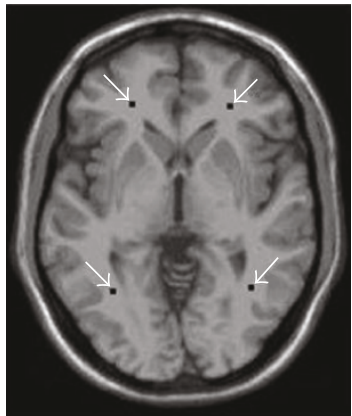

WM

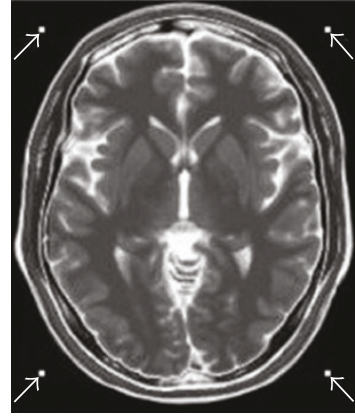

BKG

FIGURE 4: Selection of training samples for each of the four classes: WM, GM, CSF, and BKG.

successfully classified in three inconsistent ICs regardless of their appearing orders. It should be noted that we only used 20 training samples shown in Figure 4 for the three substances, WM, GM, and CSF plus the image background.

Finally, comparing the FLDA and SVM alone was also applied to the image cube formed by the three MR images in Figure 2 without an ICA transform where the same sets of training samples used for the above experiments were also used in this case. In particular, the SVM was implemented using three different kernel: linear, polynomial, and radial-based functions (RBFs). Figures 8(a) and 8(b) show the FLDA and SVM-classification results of the GM, WM, and CSF where the FLDA classification results seemed to be better than those produced by the SVM with different kernels. Nevertheless, the results in Figure 8 were still not as good as the results in Figures 5(b) and 5(c), 6(b) and 6(c), and $7(\mathrm{~b})$ and $7(\mathrm{c})$.

The above three experiments clearly demonstrated the advantages and benefits of the ICA in conjunction with a feature extraction-based classifier such as FLDA and SVM which can remedy the drawbacks resulting from the use of random initial projection vectors as well as insufficient numbers of MR images.

As a final comment, a remark on the SVM is noteworthy. One disadvantage of using the SVM is to select appropriate parameters to make it effective. Figure 9 shows an example produced by the SVM alone using a different set parameters, cost $=0.0313$ and gamma $=4$, as opposed to the parameter set, cost $=1$ and gamma $=0.5$, used in Figure $8(b)$.

Comparing Figure 9 to Figure 8(b), we immediately found that the results in Figure 9 improved significantly over the results in Figure 8(b). This example simply demonstrated that like the ICA, which suffers from instability caused by random initial conditions, the SVM also suffers from a drawback that is appropriate selection of parameters. Nevertheless, according to our experiments, if the ICA is jointly implemented with SVM, this issue can be largely alleviated. In other words, including the ICA as a preprocessing, the sensitivity to parameters used by the SVM can be greatly reduced. It should be noted that in all experiments conducted in this paper the parameters used for the SVM were fixed at cost =
0.0313 and gamma $=4$ throughout implementations including the SVM implemented in conjunction with the ICA.

\subsection{Quantitative analysis}

One great advantage of using the web images is to allow us to conduct quantitative analysis for proposed techniques. According to Figure 3, there are also other brain tissue substances such as skin, fat, glial matter, and background that also constitute different classes. However, from a clinical point of view, only the GM, WM, and CSF are of major interest. Therefore, the MRI quantitative analysis performed in this section was conducted based on contrast enhancement of these three brain tissues in the same way that was done in [18]. In this case, all tissues other than the GM, WM, and CSF were considered as a single class labeled by the background (BKG). However, it should be noted that only the GM and WM were considered and the CSF was not included for analysis in [18]. The difficulty of analyzing the CSF in [18] may have resulted from the inability of UC-ICA in dealing with insufficient MR band images.

In order to perform quantitative analysis, a quantification measure, called Tanimoto Index (TI) defined for multispectral MR images in $[23,24]$ as

$$
\mathrm{TI}=\frac{|A \cap B|}{|A \cup B|},
$$

can be used for this purpose, where $A$ and $B$ are two data sets and $|X|$ is the size of a set $X$. According to (13), TI $=0$ implies that both data sets, $A$ and $B$, are completely different and TI $=$ 1 indicates that both data sets, $A$ and $B$, are the same set. Tables 1 tabulates quantification results of GM, WM, and CSF using ICA in conjunction with classifiers FLDA and SVM in Figures 5-7, and Table 2 tabulates quantification results of GM, WM, and CSF using classifiers FLDA and SVM alone in Figure 8, where TI was the criterion specified by (13).

The "rf" in Tables 1-2 indicates the intensity nonuniformitydefined in [22]. It should be noted that the quantitative results of using ICA alone are not included because the ICA produced real values for its ICs which require an appropriate thresholding technique for quantification. A comparison 
TABLE 1: Quantification results of GM, WM, and CSF using ICA in conjunction with classifiers FLDA and SVM.

\begin{tabular}{lccccccccccccc}
\hline \multirow{2}{*}{ TI } & \multicolumn{3}{c}{ ICA + FLDA } & \multicolumn{3}{c}{ ICA + SVM (RBF kernel) } & \multicolumn{3}{c}{ ICA + SVM (linear kernel) } & \multicolumn{3}{c}{ ICA + SVM (poly kernel) } \\
& CSF & GM & WM & CSF & GM & WM & CSF & GM & WM & CSF & GM & WM \\
\hline Noise0_rf0 & 0.446 & 0.652 & 0.775 & 0.450 & 0.643 & 0.771 & 0.448 & 0.649 & 0.755 & 0.414 & 0.643 \\
Noise1_rf0 & 0.437 & 0.638 & 0.755 & 0.440 & 0.622 & 0.751 & 0.433 & 0.634 & 0.737 & 0.406 & 0.638 & 0.771 \\
Noise3_rf0 & 0.427 & 0.576 & 0.691 & 0.423 & 0.600 & 0.686 & 0.371 & 0.520 & 0.619 & 0.380 & 0.568 & 0.675 \\
Noise5_rf0 & 0.412 & 0.507 & 0.601 & 0.442 & 0.562 & 0.628 & 0.379 & 0.386 & 0.523 & 0.367 & 0.544 & 0.616 \\
\hline Noise1_rf20 & 0.429 & 0.605 & 0.736 & 0.436 & 0.638 & 0.656 & 0.367 & 0.615 & 0.730 & 0.394 & 0.604 & 0.744 \\
Noise3_rf20 & 0.451 & 0.534 & 0.693 & 0.384 & 0.573 & 0.553 & 0.299 & 0.518 & 0.536 & 0.398 & 0.560 & 0.678 \\
Noise5_rf20 & 0.427 & 0.520 & 0.587 & 0.398 & 0.610 & 0.599 & 0.368 & 0.445 & 0.458 & 0.360 & 0.504 & 0.573 \\
\hline
\end{tabular}

TABLE 2: Quantification results of GM, WM, and CSF using classifiers FLDA and SVM.

\begin{tabular}{lcccccccccccc}
\hline \multirow{2}{*}{ TI } & \multicolumn{3}{c}{ FLDA } & \multicolumn{4}{c}{ SVM (RBF kernel) } & \multicolumn{3}{c}{ SVM (linear kernel) } & \multicolumn{3}{c}{ SVM (poly kernel) } \\
& CSF & GM & WM & CSF & GM & WM & CSF & GM & WM & CSF & GM & WM \\
\hline Noise0_rf0 & 0.469 & 0.648 & 0.739 & 0.077 & 0.281 & 0.337 & 0.368 & 0.722 & 0.648 & 0.275 & 0.540 & 0.599 \\
Noise1_rf0 & 0.490 & 0.602 & 0.742 & 0.033 & 0.054 & 0.261 & 0.372 & 0.728 & 0.656 & 0.448 & 0.516 & 0.629 \\
Noise3_rf0 & 0.454 & 0.619 & 0.713 & 0.009 & 0.012 & 0.026 & 0.325 & 0.519 & 0.617 & 0.315 & 0.361 & 0.427 \\
Noise5_rf0 & 0.480 & 0.579 & 0.648 & 0.076 & 0.007 & 0.009 & 0.290 & 0.461 & 0.531 & 0.360 & 0.150 & 0.384 \\
\hline Noise1_rf20 & 0.482 & 0.607 & 0.718 & 0.022 & 0.020 & 0.256 & 0.387 & 0.623 & 0.730 & 0.358 & 0.491 & 0.603 \\
Noise3_rf20 & 0.458 & 0.602 & 0.692 & 0.005 & 0.239 & 0.022 & 0.260 & 0.555 & 0.619 & 0.364 & 0.339 & 0.456 \\
Noise5_rf20 & 0.446 & 0.582 & 0.690 & 0.053 & 0.160 & 0.276 & 0.502 & 0.408 & 0.442 & 0.455 & 0.266 & 0.337 \\
\hline
\end{tabular}

between the results of Tables 1 and 2 immediately shows that the ICA + SVM significantly outperformed SVM alone. It is also interesting to note that there was not much improvement if the FLDA + ICA outperformed the FLDA alone. For example, in the cases of Noise0rf0, Noise1rf0, and Noise1rf20, ICA + FLDA performed better than FLDA and was otherwise for the cases of Noise3rf0, Noise5rf0, Noise3rf20, and Noise5rf20. This is mainly due to the fact that the FLDA and SVM are two different types of classifiers. While the SVM requires only a few training samples, referred to as support vectors to perform effectively, the FLDA relies on a relatively large set of training samples to constitute reliable statistics for the FLDA to perform well. Since there were not sufficient samples (only 20 training samples in Figure 4 were used) to be used for training, it is expected that the FLDA would not help much in classification which was demonstrated in Tables 1 and 2.

\subsection{Real MR brian image experiments}

In this section, we further demonstrate the utility of the ICA with a feature extraction-based classification to perform post OC-ICA processing in real experiments. The real MR brain images were actually acquired from one normal volunteer by a whole body 1.5-T MR system (Sonata, Siemens, Erlangen, Germany). The routine brain MR protocol consisted of axial spin echo T1 weighted images (T1WI; TR/TE $=400 / 9 \mathrm{~ms}$ ), T2 weighted images (T2WI; TR/TE $=4000 / 91 \mathrm{~ms})$, and PD images $(\mathrm{TR} / \mathrm{TE}=4000 / 10 \mathrm{~ms})$. Other imaging parameters included for this study were slice_thickness $=6 \mathrm{~mm}$, matrix $=$
$256 \times 256, \mathrm{FOV}=24 \mathrm{~cm}$, and NEX $=2$. To reduce head movement, sponge pads were placed on both sides of a patient's head in the head coil during examination. Figure 10 shows the obtained three MR brain images.

To implement supervised FLDA and SVM, four classes were considered, WM, GM, CSF, and image background (BKG), for classification. For each class, 20 training samples were marked by dark points in the GM, CSF, WM images and bright points in the BKG image in Figure 11. These samples were selected according to prior knowledge provided by experienced radiologists where the outside of brain skull was considered as the BKG.

Following the same experiments conducted in Section 4.1, three scenarios were also produced by the FastICA using three different sets of random initial projection vectors for images in Figure 10. The three FastICA-generated ICs for each scenario are shown in Figures 12(a), 13(a), and 14(a). Interestingly, unlike the synthetic brain images considered in the previous section, the ICs in these three scenarios looked pretty much the same except their appearing orders. It is also worth noting that IC2 in Figure 12(a), IC1 in Figure 13(a), and IC2 in Figure 14(a) were heavily mixed by the GM and CSF. The FLDA and SVM were also applied to 3-IC stacked image cubes formed by the three sets of ICs produced by Figures 12(a), 13(a), and 14(a) in these three scenarios. Their classification results for WM, GM, and CSF are also shown in Figures 12(b) and 12(c), 13(b) and 13(c), and 14(b) and 14 (c) where both classifiers used the same 20 training samples selected for each of three substances and background in Figure 11 for experiments. According to the FLDA and SVM 


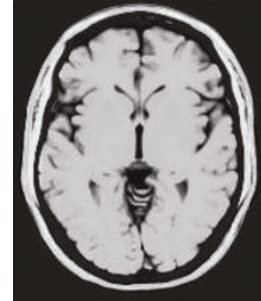

IC1

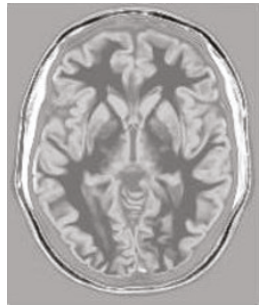

IC2

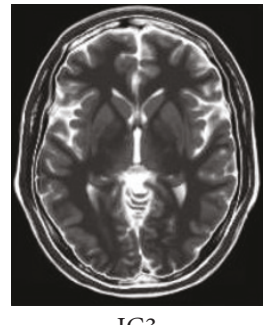

IC3 (a) Three FastICA-generated ICs

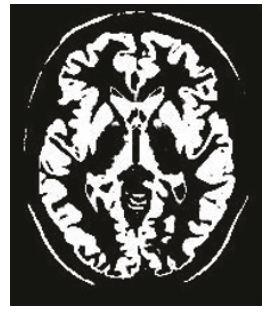

GM

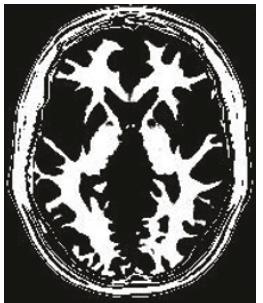

WM

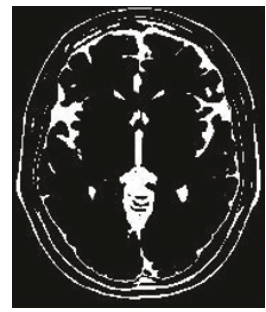

CSF (b) FLDA-classification results
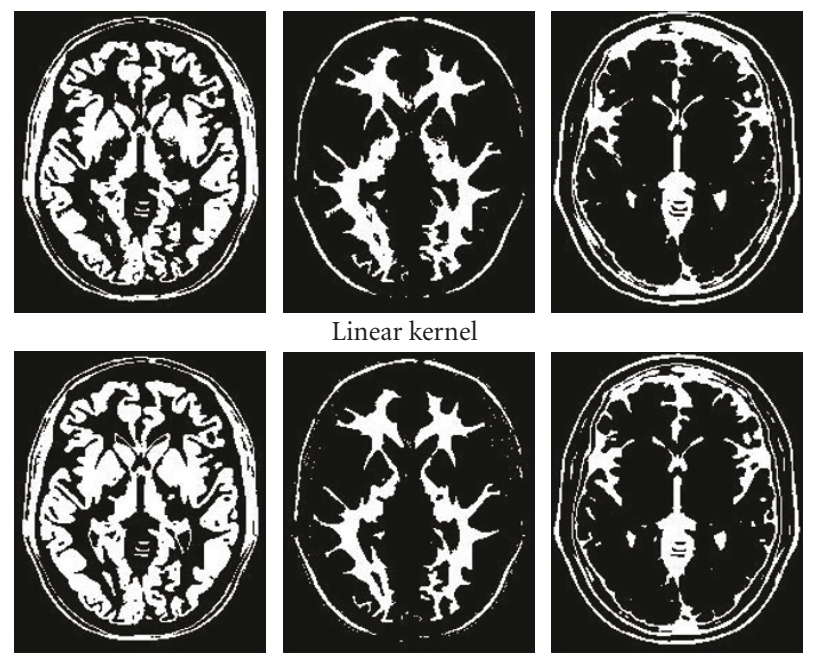

Polynomial kernel

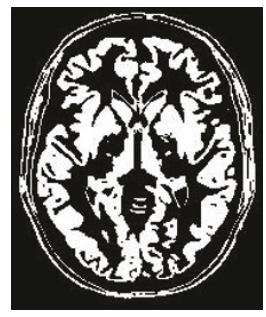

GM
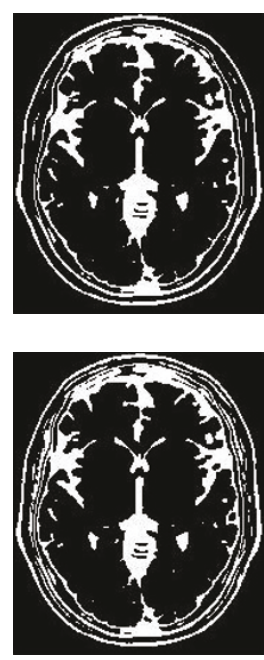

CSF

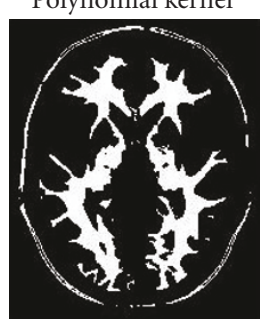

RBF kernel

WM

(c) SVM-classified ICs

FIgure 5: Scenario 1.

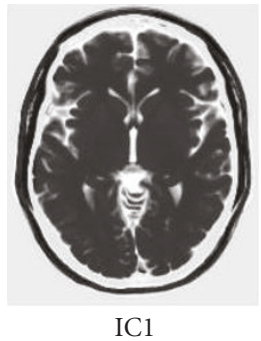

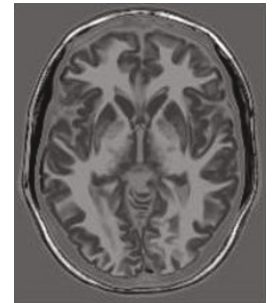

IC2

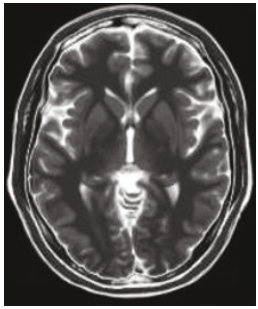

IC3 (a) Three FastICA-generated ICs

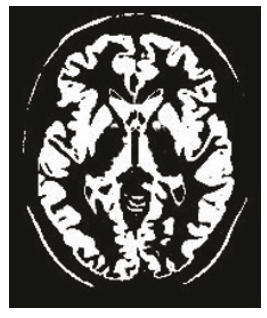

GM

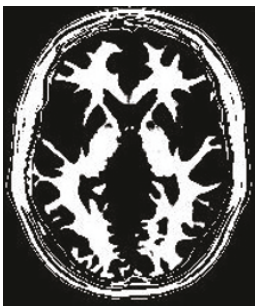

WM

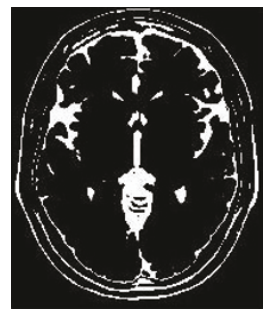

CSF (b) FLDA-classification results
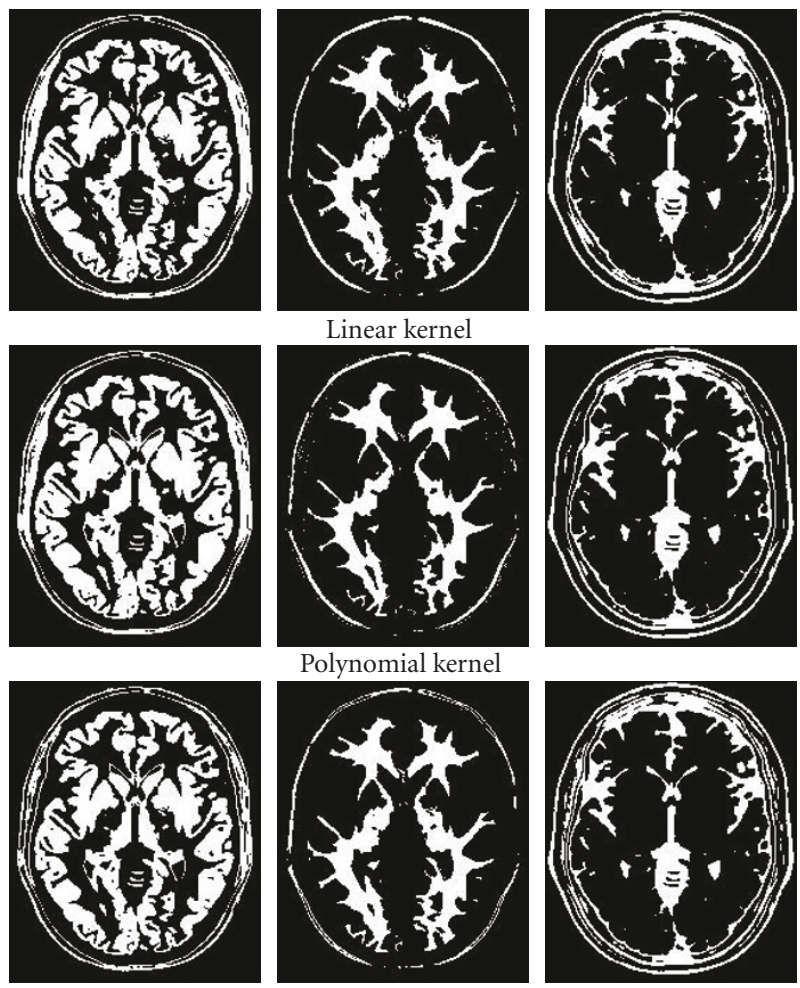

GM

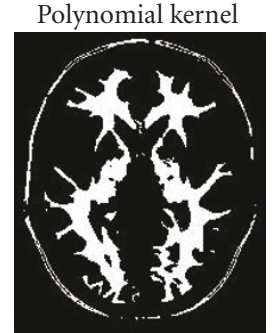

RBF kernel

WM

(c) SVM-classified ICs

Figure 6: Scenario 2.

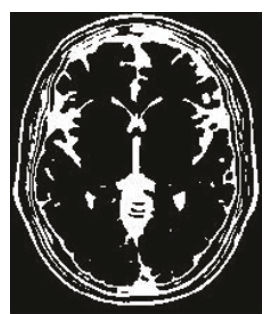

CSF

classified results, the WM, GM, and CSF were also successfully classified in each scenario.

Finally, the FLDA and SVM-classification results without using ICA are also included for comparison and results are shown in Figures 15(a)-15(b). Like experiments conducted for web synthetic brain images, the SVM was also imple-

mented with three different kernels: linear, polynomial, and radial-based functions (RBFs).

According to Figures 15(a)-15(b), using the FLDA and SVM alone without the ICA clearly performed poorly. Specifically, the results obtained by the RBF kernel were completely unrecognizable due to an inappropriate selection of 


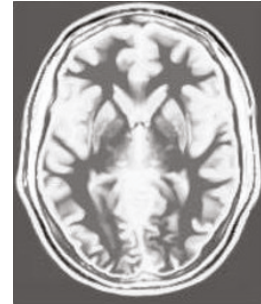

IC1

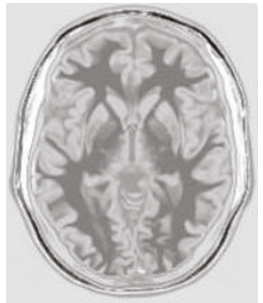

IC2

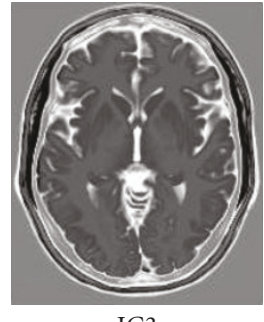

IC3 (a) Three FastICA-generated ICs

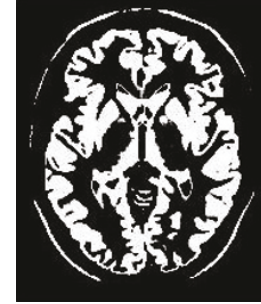

GM

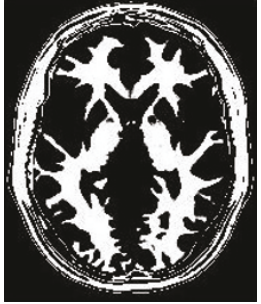

WM

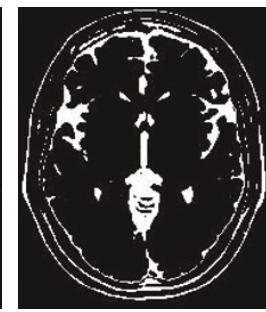

CSF (b) FLDA-classification results
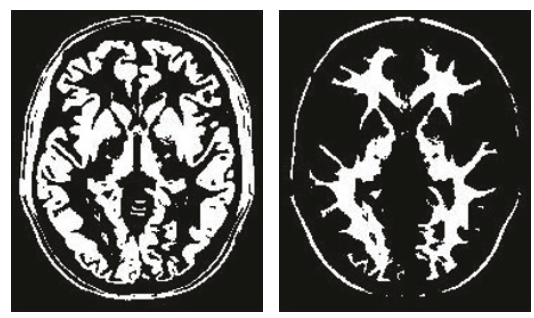

Linear kernel
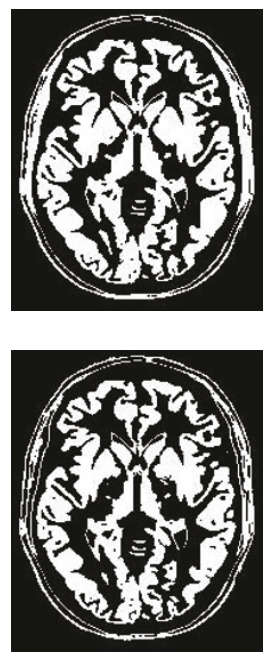

GM

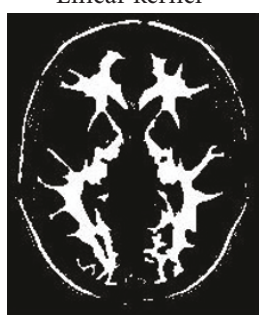

Polynomial kernel

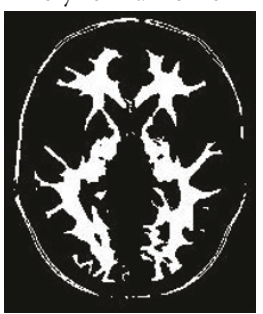

RBF kernel

WM

(c) SVM-classified ICs

Figure 7: Scenario 3.

parameters. Like Figure 9, if a different set of parameters, cost $=0.5$ and gamma $=4$, was used for the SVM with RBF kernel, the resulting classification shown in Figure 16 was significantly improved compared to the results in Figure 15(b) which used the parameters, cost $=1$ and gamma $=0.5$. Once

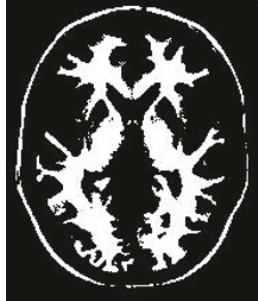

WM

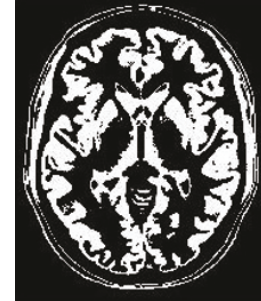

GM

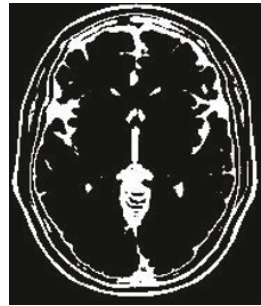

CSF (a) FLDA classification results
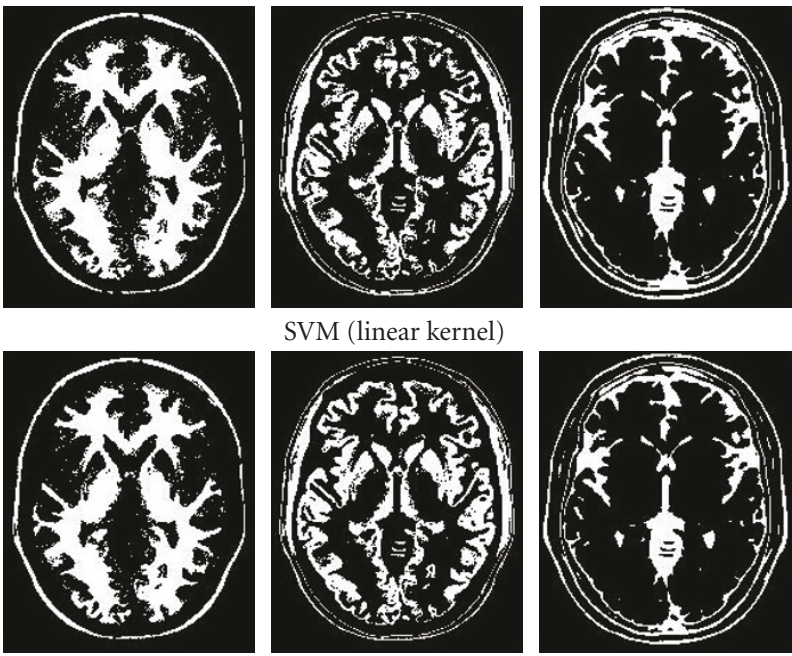

SVM (linear kernel)
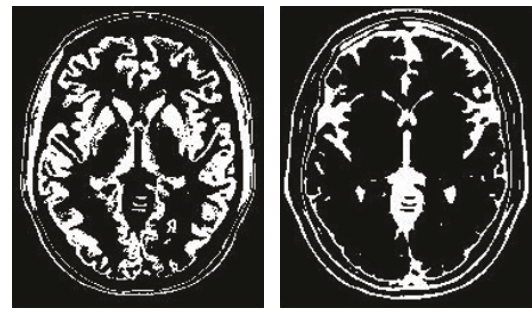

SVM (polynomial kernel)

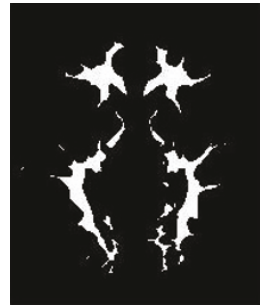

WM

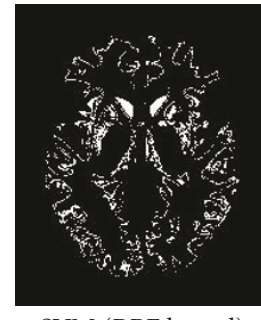

SVM (RBF kernel)

GM

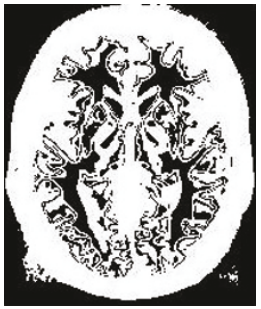

CSF (b) SVM classification results

FIGURE 8: Classification results produced by FLDA and SVM classifications.

again, this example further demonstrated instability of the SVM caused by its used parameters.

As a concluding remark, the experiments conducted in this section provide clear evidence that none of ICA, FLDA, SVM alone performed well, while their combinations, ICAFLDA and ICA-SVM, performed significantly better.

\section{DISCUSSIONS AND SUGGESTIONS}

The ICA is a versatile technique and has shown great success in many applications. However, it also presents a potential danger if this technique is blindly used without knowing its constraints and limitations. This paper provides such an example where a direct application of the ICA to MR image analysis without taking precaution may produce 
unsuccessful results. It is generally known that no more than three diagnostic pulse sequences are usually used to acquire MR images. In this case, we are limited to only three spectral band images for MR multispectral analysis and the ICA to be dealt with is actually over-complete ICA (OC-ICA) as opposed to under-complete ICA commonly used in the fMRI. Therefore, assuming that the number of sensors is greater than or equal to the number of sources to be separated, as Nakai et al. did in [18] to make the ICA under-complete, is not realistic. The experiments conducted in the previous sections clearly demonstrated serious flaws resulting from the lack of band images and the use of random initial projection vectors by an ICA algorithm. Surprisingly, these interesting issues are very important for the OC-ICA to be used as an MR multispectral image analysis technique, but have never been addressed and explored in the past. To the authors' best knowledge, this paper is believed to be the first work to investigate the utility of the OC-ICA in MR multispectral image analysis. The proposed OC-ICA coupled with a feature extraction-based classification technique as post $\mathrm{OC}$ ICA processing has yielded two major advantages. It makes use of the ICA to linearly transform three band MR images into three statistically independent component images so that these three ICA-generated independent components (ICs) can be stacked one atop another to form a new image cube which is spectrally and statistically independent in ICs. As a result, brain tissue substances that appeared in these three component images are supposed to be statistically independent or least dependent from a statistical point of view and can be classified separately and individually to avoid potential confusion that may be caused by correlation among these substances when MR images processed an image cube as a whole without an ICA transform. The clear evidence of this advantage was witnessed in our experiments. This approach is quite different from the commonly used principal components analysis (PCA) transform [25] which can only decorrelate second-order statistics that generally characterize image background rather than brain tissues which are most likely to be captured by high-order statistics. Since there are no sufficient band images to accommodate many different brain tissue substances, a single ICA-generated IC may contain more than one substance. In order to resolve this problem, a feature extraction-based classification technique is then applied to perform image analysis. This approach is also different from feature space-based techniques such as eigenimaging filter [4-9], FLDA, [19] and SVM [20, 21] which directly perform feature extraction for image analysis without using any preprocessing such as ICA transform. An advantage of our approach is to break up MR image analysis into two stage processes: the ICA in the first stage to separate distinct objects into ICs in the sense of statistical independency, then followed by a feature extraction-based classification technique in the second stage to perform target substance discrimination compared to previous approaches which extract features directly from MR images in one-shot operation. This was demonstrated in our experiments where the WM, GM, and CSF are generally captured in three separate ICs by the FastICA in the first stage. Since other substances may be also mixed with these three brain tissue sub-

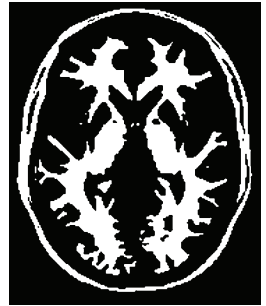

WM

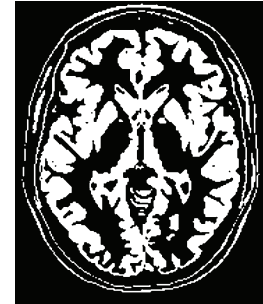

GM

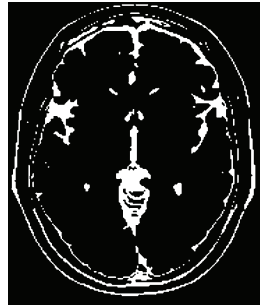

CSF
FIGURE 9: Classification results produced by SVM classification using RBF kernel.

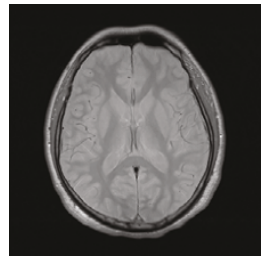

PD image

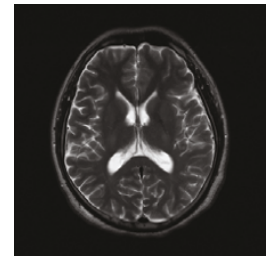

T2WI

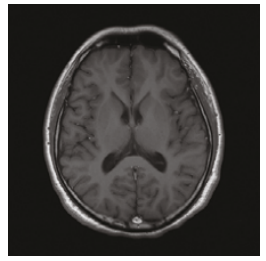

T1WI
Figure 10: Real images.

stances in different ICs, a feature extraction technique-based classification such as FLDA or SVM is then implemented in the second stage to segment our desired WM, GM, and CSF from other brain tissue substances. The experimental results demonstrated that such a two-stage process outperformed the use of the ICA or feature extraction-based classification alone.

A final comment on training samples is noteworthy. The training samples used in our experiments were supervised and selected by radiologists. In image processing, we generally do not have ideas about images to be processed. In this case, unsupervised classification is usually desirable. However, this may not be the case for MR image analysis due to the following reasons. One is that the images to be processed are brain MR images in which case the brain anatomy can be always used as prior knowledge and also as a base to select reasonably good training samples since the locations of the desired brain substances, GM, WM, CSF, can be identified $a$ priori. Another reason is that our techniques are basically developed for the use by radiologists who have been trained to be familiar with brain anatomy. Therefore, using such prior knowledge to select training samples seems very logical and natural because this can be done by radiologists themselves. A third reason is that we also have explored and conducted experiments using some unsupervised methods such as ISODATA. The results were rather poor and below an acceptance level. In this case, there is little value to include these results. This is mainly due to the fact that the brain has so many unknown substances in addition to 10 substances identified in Figure 3. It is nearly impossible to determine a reliable number of classes of interest which is a key issue in unsupervised classification. Besides, once the class number is determined, another challenging task is to find its respective training samples. This will be well beyond the scope of this paper. 


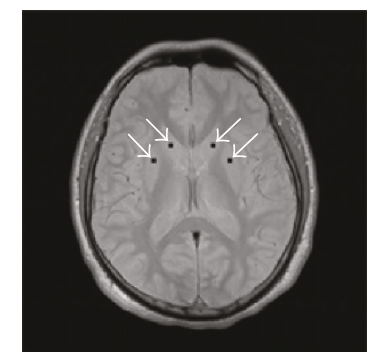

GM

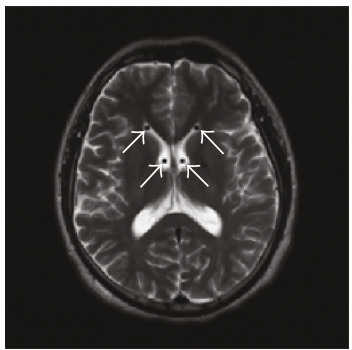

CSF

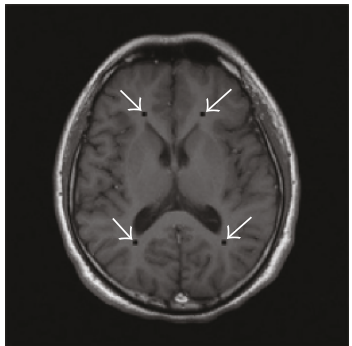

WM

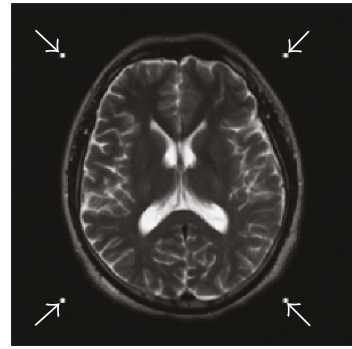

BKG

FIGURE 11: Selection of training samples for each of the four classes WM, GM, CSF and BKG.

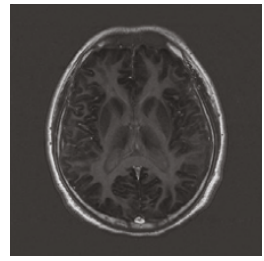

IC1

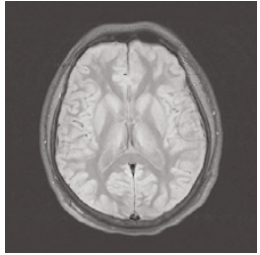

IC2

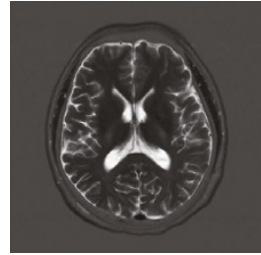

IC3

(a) Three FastICA-generated ICs

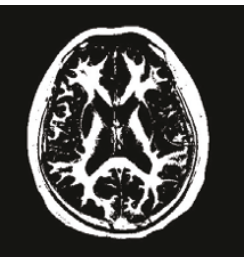

WM

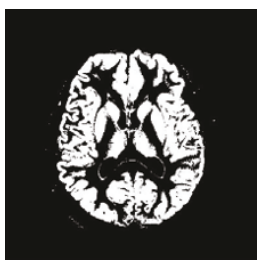

GM

(b) FLDA-classified ICs
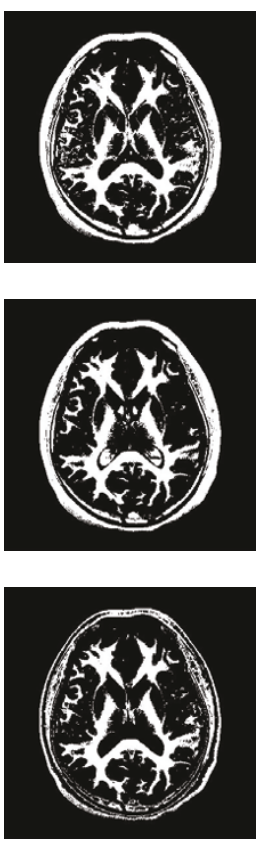

WM

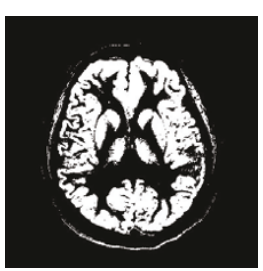

Linear kernel

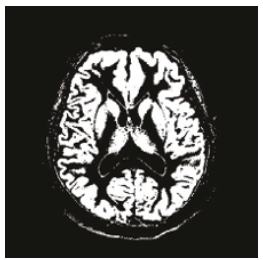

Polynomial kernel

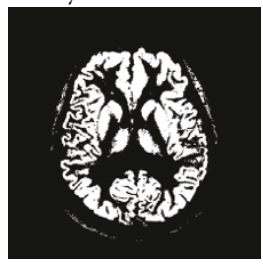

RBF kernel

GM

(c) SVM-classified ICs

Figure 12: Scenario 1.

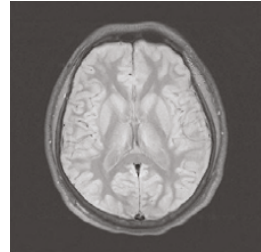

IC1

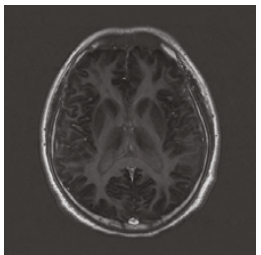

IC2

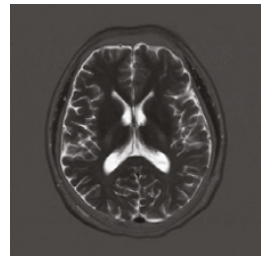

IC3

(a) Three FastICA-generated ICs

CSF
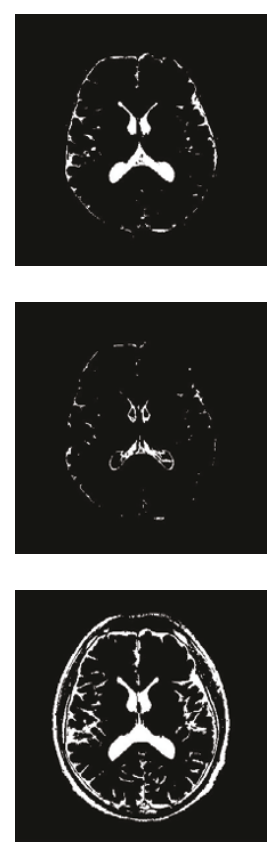

CSF

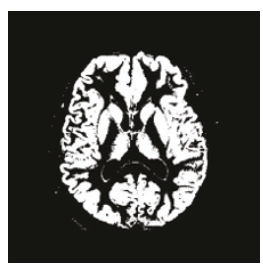

GM

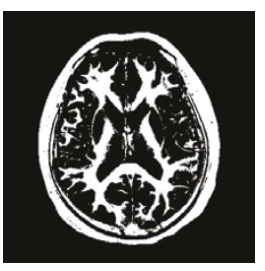

WM

(b) FLDA-classified ICs

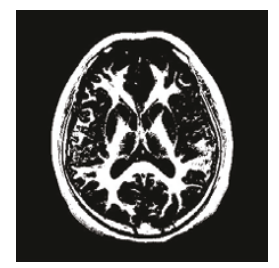

Linear kernel
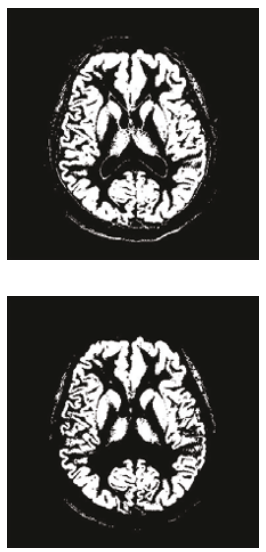

GM

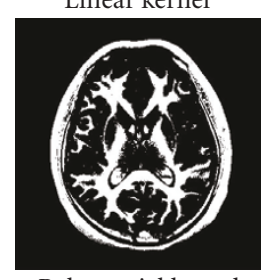

Polynomial kernel

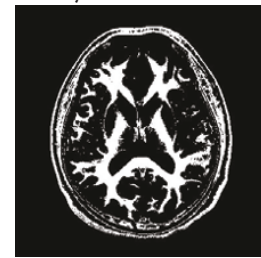

RBF kernel

WM

(c) SVM-classified ICs

Figure 13: Scenario 2.

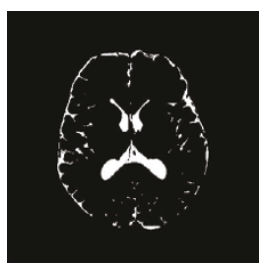

CSF
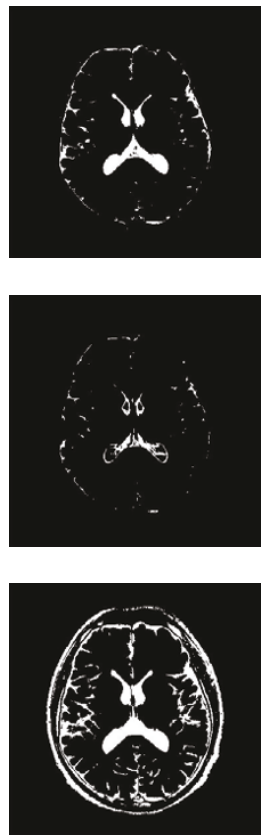

CSF . 


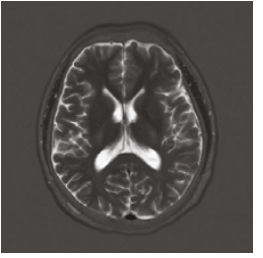

IC1

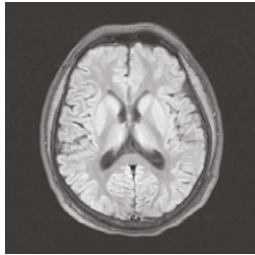

IC2

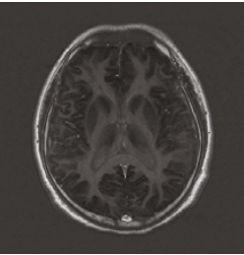

IC3 (a) Three FastICA-generated ICs

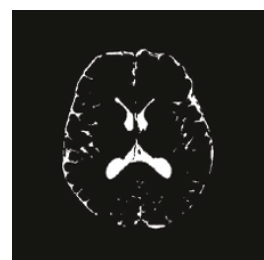

CSF

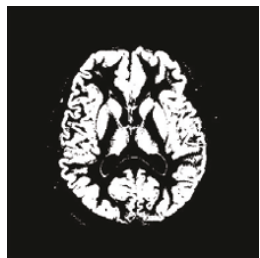

GM

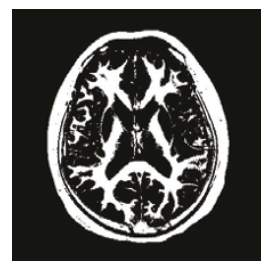

WM (b) FLDA-classified ICs
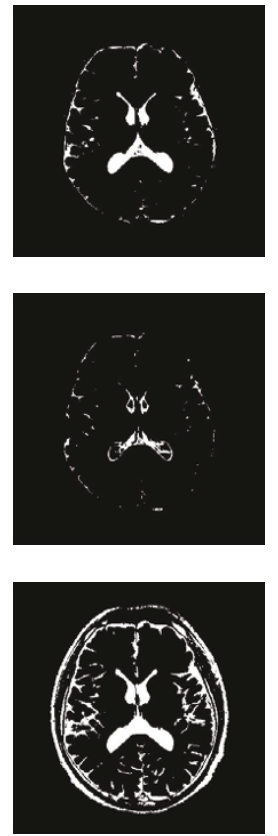

CSF

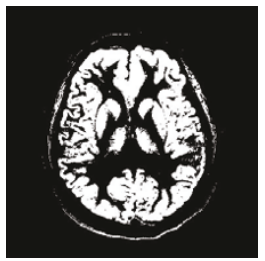

Linear kernel

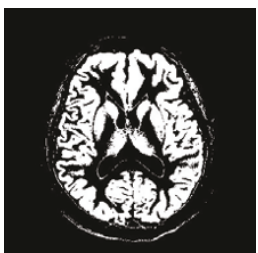

Polynomial kernel

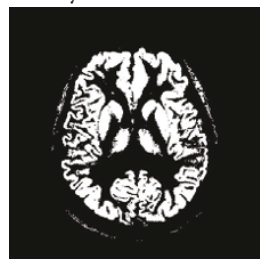

RBF kernel

GM
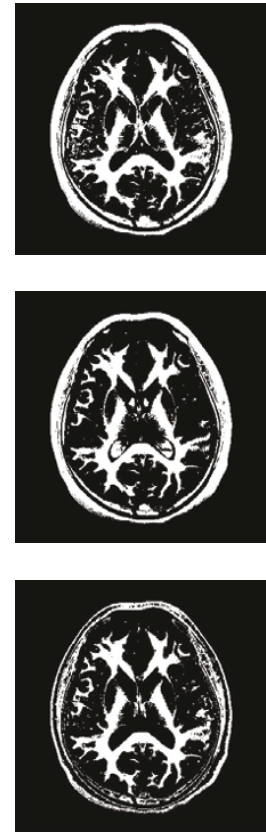

WM

(c) SVM-classified ICs

FIgURE 14: Scenario 3.

Nevertheless, our proposed technique is somewhat inbetween supervised and unsupervised classification. In other words, it uses ICA as an unsupervised technique to separate brain substances into three independent components. It is then followed by a supervised classifier either FLDA or SVM which performs classification with GM, WM, and CSF designated as desired targets to be classified, while considering all other substances as background that can be suppressed by the classifier. Accordingly, we believe that our proposed technique is the best compromise between using supervised classification alone such as FLDA and SVM, and unsupervised technique alone such as ICA.

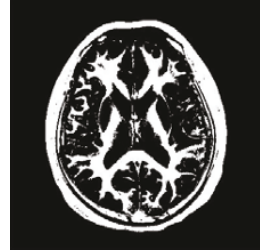

WM

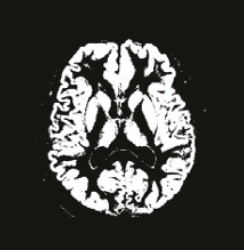

GM

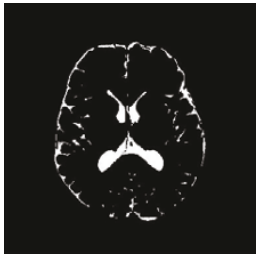

CSF (a) FLDA classification results
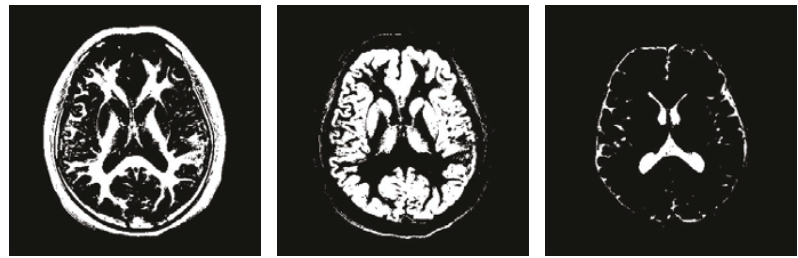

Linear kernel
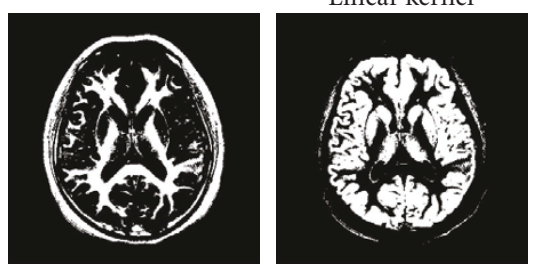

Polynomial kernel

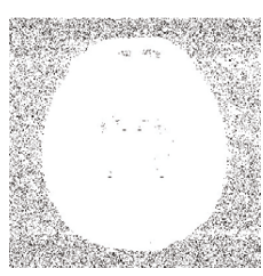

WM

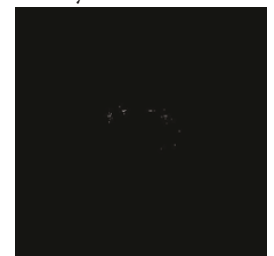

RBF kernel

GM
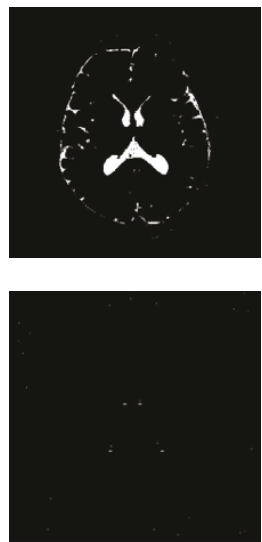

CSF

(b) SVM classification results

FIgURE 15: Classification results produced by FLDA and SVM.

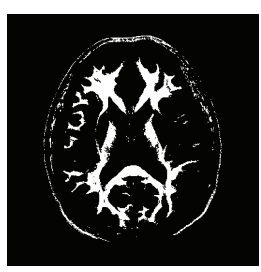

WM

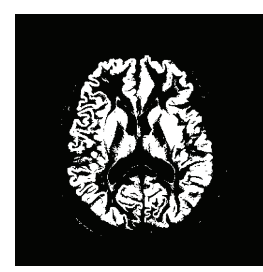

GM

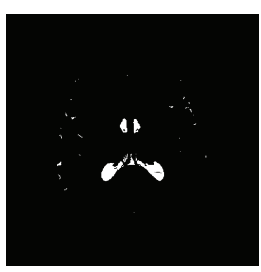

CSF
FIGURE 16: Classification results produced by SVM classification using RBF kernel.

\section{CONCLUSIONS}

This paper explores an application of the over-complete ICA (OC-ICA) to MR image analysis and investigates two major issues arising in the OC-ICA. One is due to a limited number of MR image sequences so that more than one substance of interest may be mixed and accommodated in a single IC. Another is caused by the use of random initial projection vectors. Both result in inconsistent independent components (ICs). In order to cope with these dilemmas, two feature 
extraction-based classification techniques, Fisher's discriminant analysis (FLDA) and support vector machine (SVM), are introduced to be implemented in conjunction with the ICA as post OC-ICA processing to classify substances of interest. As a result, despite that the inherent nature of OC-ICA produces inconsistent ICs, the follow-up classification is able to remedy this drawback. Most importantly, the experiments show that none of feature extraction-based classification and ICA alone can perform well, but their combination can significantly improve their performance in classification.

\section{REFERENCES}

[1] G. A. Wright, "Magnetic resonance imaging," IEEE Signal Processing Magazine, vol. 14, no. 1, pp. 56-66, 1997.

[2] L. P. Clarke, R. P. Velthuizen, M. A. Camacho, et al., "MRI segmentation: methods and applications," Magnetic Resonance Imaging, vol. 13, no. 3, pp. 343-368, 1995.

[3] J. C. Bezdek, L. O. Hall, and L. P. Clarke, "Review of MR image segmentation techniques using pattern recognition," Medical Physics, vol. 20, no. 4, pp. 1033-1048, 1993.

[4] J. P. Windham, M. A. Abd-Allah, D. A. Reimann, J. W. Froelich, and A. M. Haggar, "Eigenimage filtering in MR imaging," Journal of Computer Assisted Tomography, vol. 12, no. 1, pp. 1-9, 1988.

[5] A. M. Haggar, J. P. Windham, D. A. Reimann, D. O. Hearshen, and J. W. Froehich, "Eigenimage filtering in MR imagine: an application in the abnormal chest wall," Magnetic Resonance in Medicine, vol. 11, no. 1, pp. 85-97, 1989.

[6] H. Soltanian-Zadeh and J. P. Windham, "Novel and general approach to linear filter designed for contrast-to-noise ratio enhancement of magnetic resonance images with multiple interfering features in the scene," Journal of Electronic Imaging, vol. 1, no. 2, pp. 171-182, 1992.

[7] H. Soltanian-Zadeh, J. P. Windham, D. J. Peck, and A. E. Yagle, "A comparative analysis of several transformations for enhancement and segmentation of magnetic resonance image scene sequences," IEEE Transactions on Medical Imaging, vol. 11, no. 3, pp. 302-318, 1992.

[8] H. Soltanian-Zadeh, R. Saigal, J. P. Windham, A. E. Yagle, and D. O. Hearshen, "Optimization of MRI protocols and pulse sequence parameters for eigenimage filtering," IEEE Transactions on Medical Imaging, vol. 13, no. 1, pp. 161-175, 1994.

[9] H. Soltanian-Zadeh, J. P. Windham, and D. J. Peck, "Optimal linear transformation for MRI feature extraction," IEEE Transactions on Medical Imaging, vol. 15, no. 6, pp. 749-767, 1996.

[10] M. W. Vannier, R. L. Butterfield, D. Jordan, W. A. Murphy, R. G. Levitt, and M. Gado, "Multispectral analysis of magnetic resonance images," Radiology, vol. 154, no. 1, pp. 221-224, 1985.

[11] M. W. Vannier, T. K. Pilgram, C. M. Speidel, L. R. Neumann, D. L. Rickman, and L. D. Schertz, "Validation of magnetic resonance imaging (MRI) multispectral tissue classification," Computerized Medical Imaging and Graphics, vol. 15, no. 4, pp. 217-223, 1991.

[12] T. Taxt and A. Lundervold, "Multispectral analysis of the brain using magnetic resonance imaging," IEEE Transactions on Medical Imaging, vol. 13, no. 3, pp. 470-481, 1994.

[13] H. S. Choi, D. R. Haynor, and Y. Kim, "Partial volume tissue classification of multichannel magnetic resonance imagesa mixel model," IEEE Transactions on Medical Imaging, vol. 10, no. 3, pp. 395-407, 1991.
[14] C.-M. Wang, S.-C. Yang, P.-C. Chung, et al., "Orthogonal subspace projection-based approaches to classification of MR image sequences," Computerized Medical Imaging and Graphics, vol. 25, no. 6, pp. 465-476, 2001.

[15] C.-M. Wang, C.-C. Chen, S.-C. Yang, et al., "An unsupervised orthogonal subspace projection approach to MR image classification MR images for classification," Optical Engineering, vol. 41, no. 7, pp. 1546-1557, 2002.

[16] C.-M. Wang, C.-C. Chen, Y.-N. Chung, et al., "Detection of spectral signatures in MR images for classification," IEEE Transactions on Medical Imaging, vol. 22, no. 1, pp. 50-61, 2003.

[17] A. Hyvarinen, J. Karhunen, and E. Oja, Independent Component Analysis, John Wiley \& Sons, New York, NY, USA, 2001.

[18] T. Nakai, S. Muraki, E. Bagarinao, et al., "Application of independent component analysis to magnetic resonance imaging for enhancing the contrast of gray and white matter," $\mathrm{Neu}$ roImage, vol. 21, no. 1, pp. 251-260, 2004.

[19] R. O. Duda and P. O. Hart, Pattern Classification and Scene Analysis, John Wiley \& Sons, New York, NY, USA, 1973.

[20] S. Haykin, Neural Networks: A Comprehensive Foundation, chapter 6, Prentice-Hall, Upper Saddle River, NJ, USA, 2nd edition, 1999.

[21] V. N. Vapnik, Statistical Learning Theory, John Wiley \& Sons, New York, NY, USA, 1998.

[22] http://www.bic.mni.mcgill.ca/brainweb/faq.html\#protocols.

[23] S. Theodoridis and K. Koutroumbas, Pattern Recognition, Academic Press, New York, NY, USA, 1999.

[24] R. Valdes-Cristerna, V. Medina Banuelos, and O. YanezSuarez, "Coupling of radial-basis networks and active contour model for multispectral brain MR images," IEEE Transactions on Biomedical Engineering, vol. 51, no. 3, pp. 459-470, 2004.

[25] H. Grahn, N. M. Szeverenyi, M. W. Roggenbuck, F. Delaglio, and P. Geladi, "Data analysis of multivariate magnetic resonance images I. A principal component analysis approach," Chemometrics and Intelligent Laboratory Systems, vol. 5, pp. 311-322, 1989. 